\section{MS39-P3 From Micromolecules' to Macromolecules' Structural Dynamics Properties: Ultrafast Chiroscopy with Synchrotron and Free Electron Laser Radiation}

Simone A. Techert ${ }^{1}$, Sreevidya Thekku-Veedu ${ }^{1}$, Sadia Bari ${ }^{1}$, Rebecca Boll ${ }^{1}$, Zhong Yin ${ }^{1}$, Darina Storozhuk ${ }^{1}$, Philipp Busse ${ }^{2}$, Dirk Raiser ${ }^{2}$

\section{DESY}

2. MPIbpC

email: simone.techert@desy.de

Common for all time-resolved $\mathrm{x}$-ray experiments is the applied pump / probe scheme, where an optical pump-laser initiates a reaction whose structural time evolution is then investigated by $\mathrm{x}$-ray probe pulses at various time delays. X-ray photon-in / photon-out techniques are based on diffraction or spectroscopic techniques like near edge spectroscopy or x-ray emission spectroscopy. Meanwhile x-ray spectroscopic techniques probe the local environment around specific atoms in a molecule - such as orbitals, diffraction studies reveal the structure of the bulk of periodic systems. In the present contribution we will give an overview of our strategy utilizing the pulsed characteristics of X-ray sources, in particular synchrotrons and free electron lasers, to gain structural dynamics information of micro- and macromolecules on their time scales of reactivity which ranges from femtoseconds to milliseconds. Method wise, Chiroscopy will be introduced and discussed in the context of time-resolved X-ray diffraction / X-ray scattering and X-ray spectroscopy. In a new concept of photon-assisted reaction trigger we will introduce an experimental strategy to overcome the paradigm of optical light initiation for time-resolved X-ray scattering experiments and what it means for chiroscopy. Finally, we will summarize our efforts in systematizing the characteristic structural changes in molecular systems during chemical reactions to some kind of "periodic table" of structural dynamics allowing predicting reaction properties in chemistry and biochemistry from a time-dependent structural point of view.

References: S. Bari, R. Boll, S. E. Canton, L. Glaser, K. Idzik, K. Kubicek, D. Raiser, S. Thekku Veedu, Z. Yin, $\mathrm{S}$. Techert, High flux X-ray sources and Free Electron Lasers for studying ultrafast time structure imprints in complex chemical and biochemical reactions, in: X-ray Free Electron Lasers, eds. U. Bergmann, P. Pellegrini, Oxford University Press, in press (2016) and references therein.

Keywords: time-resolved x-ray crystallography
MS40 New detectors for high

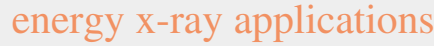

Chairs: Heinz Graafsma, Lothar Strüder
MS40-P1 3D energy dispersive detector (pnCCD) for ultra-hard x-rays Laue diffraction: In situ analysis of lattice deformation in polycrystalline Copper

Ali Abboud ${ }^{1}$, Jozef Keckes ${ }^{2,3}$, Mohammad Shokr ${ }^{1}$, Amir Tosson ${ }^{1}$, Lothar Strüder ${ }^{4}$, Manuela Klaus ${ }^{5}$, Chritopher Klaus ${ }^{5}$, Ullrich Pietsch $^{1}$

1. Department of Physics, University of Siegen, Siegen, Germany

2. Montanuniversität Leoben, Leoben, Austria

3. Material Center Leoben Forschungs GmbH, Leoben, Austria

4. PNSensor GmbH, Munich, Germany

5. Helmholtz-Zentrum Berlin for Materials and Energy, Berlin, Germany

email: aliabbouds@hotmail.com

The deformation behavior of individual grains in a large grained polycrystalline $\mathrm{Cu}$ sample is studied during in situ tensile loading. The Laue diffraction experiment was carried out at the EDDI beamline (@ Bessy II) using ultra-hard $\mathrm{x}$-rays (5 to $160 \mathrm{keV}$ ) and a 3D energy dispersive pnCCD detector. The recorded Laue-patterns as a function of tensile loading shows the evolution of spatially well-defined Laue spots into streaking ones. The pnCCD was used to record the position and the energy spectrum of each diffraction peak in all of the collected Laue patterns in a single shot experiment, which allowed to index all Laue spots and assign them to individual grains simultaneously. The two-fold information yields the crystal cell orientation of each individual grain and its relative orientation with respect to the surrounding grains, i.e. the texture of the probed region. Results show that the grains rotate around the loading axis until the preferred slip direction is aligned with the tensile direction. This phenomena is accompanied by a continuous expansion of the lattice parameters followed by a rapid strain release resulting in internal stress relaxation, while the strain increases again at very high strain levels. In addition, the energy profile of the elongated Laue spots shows that in most cases rotational strain was the man response of the grains with respect to external strain.

Keywords: hard x-rays, pnccd, polycrystalline, copper, in-situ energy dispersive $\mathrm{x}$-ray diffraction 